\title{
Increased Interleukin-6 Levels in the Astrocyte-Derived Exosomes of Sporadic Amyotrophic Lateral Sclerosis Patients
}

\author{
Yong Chen ${ }^{1}$, Kailin Xia ${ }^{1}$, Lu Chen ${ }^{1}$ and Dongsheng Fan ${ }^{1,2 *}$ \\ ${ }^{1}$ Department of Neurology, Peking University Third Hospital, Beijing, China, ${ }^{2}$ Key Laboratory for Neuroscience, Ministry \\ of Education/National Health Commission, Peking University, Beijing, China
}

\section{OPEN ACCESS}

Edited by:

Pierre-francois Pradat, Hôpitaux Universitaires Pitié

Salpêtrière, France

Reviewed by:

Stephanie Marie-Rose Duguez, Ulster University, United Kingdom

Olivier Lucas,

INSERM U1051 Institut des Neurosciences de Montpellier, France

${ }^{*}$ Correspondence: Dongsheng Fan dsfan2010@aliyun.com

Specialty section:

This article was submitted to Neurodegeneration, a section of the journal

Frontiers in Neuroscience

Received: 17 February 2019

Accepted: 20 May 2019

Published: 05 June 2019

Citation:

Chen Y, Xia K, Chen L and Fan D (2019) Increased Interleukin-6 Levels in the Astrocyte-Derived Exosomes of Sporadic Amyotrophic Lateral

Sclerosis Patients.

Front. Neurosci. 13:574. doi: 10.3389/fnins.2019.00574
Neuroinflammation plays an important role in amyotrophic lateral sclerosis (ALS) pathogenesis. However, it is difficult to evaluate inflammation of the central nervous system (CNS) or the relationship between neuroinflammation and disease progression in ALS patients. Recent advances in the field of exosomes and CNS-derived exosomes extraction technology provide the possibility of measuring the inflammatory status in the CNS without brain biopsy. In this pilot study, we extracted astrocyte-derived exosomes from the plasma of sporadic ALS patients and age-, sex-matched healthy controls and determined Interleukin-6 (IL-6) levels by an enzyme-linked immunosorbent assay (ELISA). The IL-6 levels in astrocyte-derived exosomes were increased in sALS patients and positively associated with the rate of disease progression. However, the association between IL-6 levels and disease progression rate was limited to patients whose disease duration were less than 12 months. These data suggest an increased inflammatory cascade in the CNS of sALS patients. Our pilot study demonstrates that CNS-derived exosomes could be useful to reveal neuroinflammation of the CNS in ALS patients.

Keywords: amyotrophic lateral sclerosis, astrocytes, exosomes, disease progress, interleukin-6

\section{INTRODUCTION}

Amyotrophic lateral sclerosis (ALS) is a rare, progressive neurodegenerative disease that affects upper and lower motor neurons and leads to fatal paralysis (Brown and Al-Chalabi, 2017). Ultimately, most ALS patients die within 3-5 years after disease onset due to respiratory failure. Approximately $90-95 \%$ of ALS cases are the sporadic type (sALS), and the remaining cases are the familial type (fALS). To date, more than 20 genes that cause fALS and sALS have been identified (Brown and Al-Chalabi, 2017). Scientific advances in genetic studies in the ALS field have improved our understanding of ALS pathogenesis. However, the exact etiology and pathogenesis of ALS are still unknown. As a result, there is no effective treatment for the disease. Riluzole and edaravone are the only two approved drugs for the treatment of ALS, and they solely delay disease progression for several months (Kumar et al., 2016; Rothstein, 2017).

Numerous intrinsic and extrinsic factors are involved in ALS motor neuron degeneration. One possible factor involved in motor neuron degeneration in ALS is neuroinflammation. Accumulating evidence indicates that ALS patients have chronic inflammation, as demonstrated by activated microglia and astrocytes, as well as infiltration of peripheral monocytes and lymphocytes into 
the CNS (Zhao et al., 2013; Liu and Wang, 2017). Increased serum/plasma and CSF levels of some cytokines, such as tumor necrosis factor-alpha (TNF- $\alpha$ ), interleukin-6 (IL-6), IL-8, and interferon-beta (IFN- $\beta$ ), have been detected in ALS patients when compared to controls (Ono et al., 2001; Mitchell et al., 2009; Fiala et al., 2010; Mitchell et al., 2010; Italiani et al., 2014; Ehrhart et al., 2015; Liu et al., 2015; Hu et al., 2017). Beyond demonstrating ongoing inflammatory processes in ALS patients, these inflammatory biomarkers could also be used as diagnostic and prognostic biomarkers for clinical use because they have been reported to distinguish ALS from healthy or disease controls (Vu and Bowser, 2017; Gonzalez-Garza et al., 2018) and to predict the disease prognosis (Su et al., 2013; Liu et al., 2015). Activated microglia and astrocytes in the CNS play a vital role in the neuroinflammation process in ALS patients; however, the determination of the inflammatory biomarkers in serum/plasma and CSF only indirectly reflects the status of the CNS. Recently, scientific advances in the field of exosomes and CNS-derived exosome extraction technology have provided the possibility of measuring the inflammatory status in the CNS without brain biopsy.

Exosomes are approximately 30-100 nm extracellular vesicles with lipid bilayer membranes that are secreted by almost all types of cells, including neurons, microglia and astrocytes (Raposo and Stoorvogel, 2013; Yanez-Mo et al., 2015). Exosomes contain proteins, lipids and RNA and transfer them between cells. Therefore, exosomes play an important role in intercellular communication. Moreover, different cell types can secrete exosomes with different biomarkers, which could help to identify the exosome source (Beninson and Fleshner, 2014). Due to their specific characteristics, exosomes have attracted large amounts of attention in various studies ranging from mechanistic analyses to clinical research (Jarmalaviciute and Pivoriunas, 2016; Goh et al., 2017). In addition, exosomes can cross the blood-brain barrier (BBB) from both directions. As a result, CNS-derived exosomes can be detected in the blood and may help to reveal the pathophysiology of brain diseases without the use of brain biopsy and CSF analysis (Mustapic et al., 2017). In recent studies, several strategies to extract CNS-derived exosomes from peripheral blood have been reported (Mustapic et al., 2017; Kuwano et al., 2018). However, CNS-derived exosome-based studies focusing on ALS have not been previously reported.

Based on the above information, we hypothesized that inflammatory biomarkers in astrocyte-derived exosomes (ADEs) may increase and may be associated with clinical features in ALS patients. In this pilot study, we extracted ADEs from the plasma of sporadic ALS patients and age-, sex-matched healthy controls to determine the IL-6 levels in ADEs and, ultimately, we detected increased IL-6 levels in ADEs of sALS patients, which were positively associated with the rate of disease progression.

\section{PARTICIPANTS AND METHODS}

\section{Participants}

This study was approved by the Ethics Committee of the Perking University Third Hospital, Beijing, China. All ALS patients and age-, sex-matched healthy control individuals signed the informed consent before peripheral blood samples were drawn. Patients and controls were recruited from the Department of Neurology of Perking University Third Hospital. Clinically definite and probable sALS patients were diagnosed based on the EI Escorial revised criteria (Brooks et al., 2000) and further evaluated by the revised ALS functional rating scale (ALSFRS-R) (Cedarbaum et al., 1999). The rate of disease progression $(\Delta \mathrm{FS})$ was calculated as follows: $\Delta \mathrm{FS}=(48$-ALSFRS-R at "time of diagnosis")/duration from onset to diagnosis (month) (Kimura et al., 2006).

\section{Plasma Sampling in ALS Patients and Controls}

Samples containing two milliliters of peripheral blood from ALS patients and healthy control individuals were collected into EDTA tubes. To extract plasma, blood samples were centrifuged at $1500 \mathrm{~g}$ for $10 \mathrm{~min}$ to remove blood cells. Then, the supernatant was subjected to another centrifugation at $2500 \mathrm{~g}$ for $20 \mathrm{~min}$ to remove the platelets and cell debris. Finally, the plasma was stored at $-80^{\circ} \mathrm{C}$ until use.

\section{Extraction of ADEs From Plasma}

The method to extract the ADEs from plasma was modified from a previously published article (Mustapic et al., 2017). Briefly, $0.25 \mathrm{ml}$ plasma was incubated with $0.2 \mu \mathrm{l}$ thromboplastin (System Biosciences, Mountain View, CA, United States) for $5 \mathrm{~min}$. Then, $298 \mu \mathrm{l}$ calcium- and magnesium-free Dulbecco's Balanced Salt Solution $\left(\mathrm{DBS}^{-2}\right)$ was added with protease inhibitor cocktail (Roche, Indianapolis, IN) and phosphatase inhibitor cocktail (Thermo Fisher Scientific), followed by centrifugation at $10,000 \mathrm{rpm}$ for $5 \mathrm{~min}$ at $4^{\circ} \mathrm{C}$. The supernatants were harvested, followed by addition of $126 \mu l$ per tube of ExoQuick (System Biosciences, Mountain View, CA, United States). After a second centrifugation at $1500 \mathrm{~g}$ for $30 \mathrm{~min}$ at $4^{\circ} \mathrm{C}$, total exosomes were harvested by removing the supernatant. To enrich ADEs, total exosomes were resuspended in $250 \mu \mathrm{l}$ of $\mathrm{ddH}_{2} \mathrm{O}$ with protease inhibitor cocktail and phosphatase inhibitor cocktail and incubated for at least $120 \mathrm{~min}$ at $4^{\circ} \mathrm{C}$. Then, $1.5 \mu \mathrm{g}$ biotinylated mouse anti-human glutamine aspartate transporter (ACSA-1) antibody (Miltenyi Biotec, Auburn, CA, United States) in $50 \mathrm{ml}$ of 3\% bovine serum albumin (BSA; 1:3.33 dilution of Blocker BSA 10\% solution in $\mathrm{DBS}^{-2}$; Thermo Fisher Scientific) was added per tube and mixed for $60 \mathrm{~min}$ at room temperature, followed by the addition of $10 \mu \mathrm{l}$ streptavidin-agarose Ultralink resin (Thermo Fisher Scientific) in $40 \mathrm{ml} \mathrm{3 \%} \mathrm{BSA} \mathrm{and} \mathrm{incubation} \mathrm{with} \mathrm{mixing} \mathrm{for} \mathrm{another} 20 \mathrm{~min}$ at room temperature. After centrifugation at $400 \mathrm{~g}$ for $10 \mathrm{~min}$ at $4^{\circ} \mathrm{C}$, the supernatant was removed, and each pellet was suspended in $200 \mu \mathrm{l}$ cold $0.1 \mathrm{M}$ glycine- $\mathrm{HCl}(\mathrm{pH}=3.0)$ by gentle mixing for $10 \mathrm{~s}$ and centrifugation at 4,500 $\mathrm{g}$ for $5 \mathrm{~min}$. The supernatants were then harvested, and $25 \mu \mathrm{l}$ of $3 \%$ BSA and $15 \mu$ l of 1 M Tris$\mathrm{HCl}(\mathrm{pH}=8.0)$ were added. Finally, $260 \mu \mathrm{l}$ mammalian protein extraction reagent (M-PER, Thermo Fisher Scientific) was added, and the solution was mixed. The resultant $0.5 \mathrm{ml}$ lysates of ADEs were stored at $-80^{\circ} \mathrm{C}$. Evidence for enrichment of exosomes 
from neural sources in plasma has been demonstrated previously (Mustapic et al., 2017).

\section{Measurement of IL-6 Levels in ADEs and Plasma}

Astrocyte-derived exosome proteins were quantified using a single-plex high-sensitivity and high-dynamic-range ELISA for IL-6 (Rockville, MD, United States Cat\# K151AKC) (Chaturvedi et al., 2015) and by using enzyme-linked immunosorbent assay (ELISA) kits for the tetra-spanning exosome marker CD81 (Cusabio Technology, Wuhan, China), according to the suppliers' directions. The mean value for all determinations of CD81 in each assay group was set at 1.00, and the relative values for each sample were used to normalize their recovery. The plasma IL-6 levels in both groups were also measured. The protein levels were measured by board-certified laboratory technicians who were blinded to the clinical information.

To ensure the specificity of the tests, negative control groups were set up in this study. In the negative control group one, the biotinylated anti-ACSA-1 antibody was replaced with 3\% BSA. In the negative control group two, the total exosomes solution resuspended from ExoQuick pellet was replaced by ddH2O.

\section{Statistical Analyses}

Data are presented as numbers, means and standard deviations, or medians (interquartile range, IQR) as appropriate. Normal distributions of datasets were assessed by the Shapiro-Wilks test. Unpaired Student $t$-tests, $\chi^{2}$ test or one-way ANOVA, followed by Tukey analysis, were used to examine differences between groups. Pearson's correlation was used for statistical correlation analysis. The differences between groups were considered significant if the $p$-value was less than 0.05 (two-tailed). All statistical analyses and graphs were performed using GraphPad Prism 6 (GraphPad Software Inc., San Diego, United States).

\section{RESULTS}

In this pilot study, 40 ALS patients and 39 healthy controls were recruited. The detailed clinical information for these two groups are summarized in Table 1. The ALS patients and controls were comparable, as there was no difference in age or sex ratio between the two groups. Of the 40 ALS patients, 12 were bulbar onset and 28 were limber onset; 10 ALS cases were diagnosed as definite, and the remainder were probable. The median delay of diagnosis for all patients was 9.23 months. The mean ALSFRS-R score for the patients was $39.83 \pm 1.08$, and the median disease progression rate was 0.56 . The extracted ADEs were validated by western blot. The result showed that the ADEs were positive for CD63, but negative for calnexin (Supplementary Figure S1). The ADEs were also verified by transmission electron microscope (Supplementary Figure S2). In the CD81 and IL-6 test, the negative control group one and two were all at background levels. The CD81-normalized levels of IL-6 in ADEs were significantly higher in ALS patients $(40.40 \pm 2.11 \mathrm{pg} / \mathrm{ml})$ than in controls $(22.45 \pm 1.90 \mathrm{pg} / \mathrm{ml}$ ) (Figure 1A). However, among 40 ALS patients and 39 healthy controls, the IL- 6 was detectable only in 12 controls and 15 ALS patients. There was no difference in detection rate between the two groups. The plasma IL-6 levels ranged from 0.13 to $4.58 \mathrm{pg} / \mathrm{mL}$ in controls and 0.39 to $15.69 \mathrm{pg} / \mathrm{ml}$ in ALS patients (Supplementary Figure S3A). There was no difference in plasma IL-6 levels between controls and ALS patients $(p=0.3614)$ and there was no correlation between IL-6 levels in plasma and ADEs $(r=0.3384, p=0.2173$ for ALS group; $r=-0.2657, p=0.4038$ for control group; Supplementary Figures S3B,C).

The ALS patients were further divided into subgroups according to the following: onset site: bulbar onset (ALS-B) or limber onset (ALS-L); diagnosis level: definite (ALS-D) or probable (ALS-P); and disease duration: less than 12 months $(A L S<12$ ) or greater than or equal to 12 months (ALS $\geq 12$ ). As shown in Figures 1B-D, compared with the control group, the levels of IL- 6 in ADEs were increased in all ALS subgroups. However, there was no difference between the ALS subgroups.

The correlations of the levels of IL- 6 in ADEs with clinical parameters are shown in Figure 2. The IL-6 levels correlated positively with the disease progression rate $(r=0.4696, p=0.002)$. However, IL-6 levels in the ADEs of ALS patients did not correlate with total ALSFRS-R scores $(r=-0.2021, p=0.2110)$, diagnosis delay $(r=-0.1735, p=0.2845)$ or patient age $(r=-0.1087$, $p=0.5560)$. In controls, IL-6 levels also did not correlate with age (data not shown). When the patients were separated into two groups according disease duration (ALS $<12 \mathrm{~m}$ or ALS $\geq 12 \mathrm{~m}$ ), a positive correlation between IL- 6 levels and disease progression was only verified in the ALS $<12$ m group $(r=0.6605, p=0.015)$ (Figure 3A) but not in the ALS $\geq 12 \mathrm{~m}$ group $(r=0.3510$, $p=0.1291)$ (Figure 3B).

\section{DISCUSSION}

The present study demonstrated that the levels of IL-6 in ADEs of sALS patients were increased and positively associated with the rate of disease progression, especially in patients at an earlier disease stage. These data suggest that the inflammatory cascade is augmented in the CNS of sALS patients. Analysis of CNSderived exosomes in peripheral blood has recently attracted immense attention. Numerous studies have demonstrated that CNS-derived exosomes could be helpful to understand the

TABLE 1 | Characteristics of ALS patients and healthy controls.

\begin{tabular}{lccc}
\hline & Control & ALS & $\boldsymbol{p}$ \\
\hline Cases (male/female) & $39(25 / 14)$ & $40(26 / 14)$ & 1 \\
Age (mean \pm SE) & $55.74 \pm 1.32$ & $54.35 \pm 2.02$ & 0.57 \\
Onset site: bulbar/limb & NA & $12 / 28$ & NA \\
Diagnosis delay (months) & NA & $9.23(9.68)$ & NA \\
Definite/probable & NA & $10 / 30$ & NA \\
ALSFRS-R & NA & $39.83 \pm 1.08$ & NA \\
$\Delta$ FS & NA & $0.56(0.71)$ & NA \\
IL-6 (pg/ml) & $22.45 \pm 1.90$ & $40.40 \pm 2.11$ & $<0.001$ \\
\hline
\end{tabular}

Diagnosis delay, interval from the initial symptoms to diagnosis; ALSFRS-R, revised amyotrophic lateral sclerosis functional rating scale; $\triangle F S$, disease progression rate. 
A

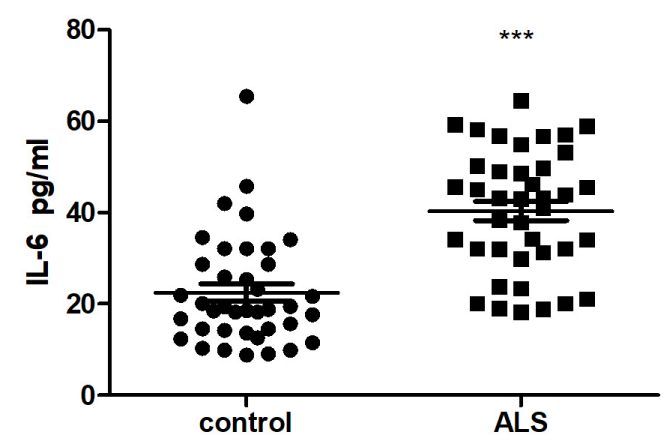

C

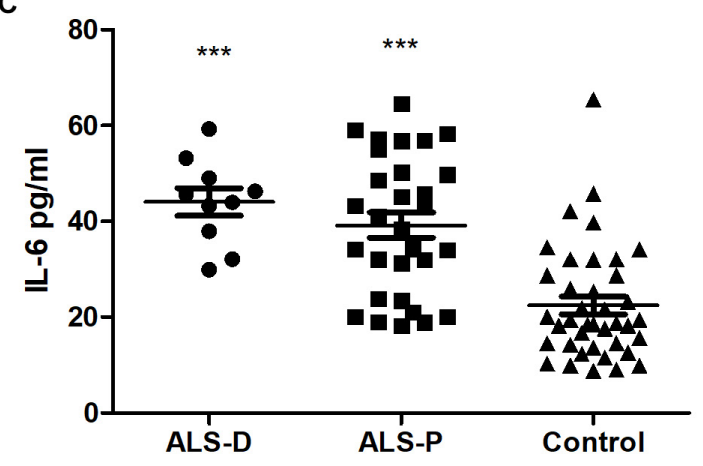

B

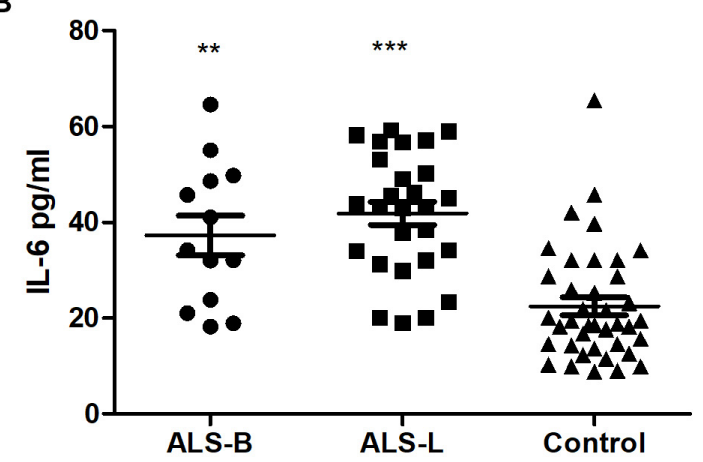

D

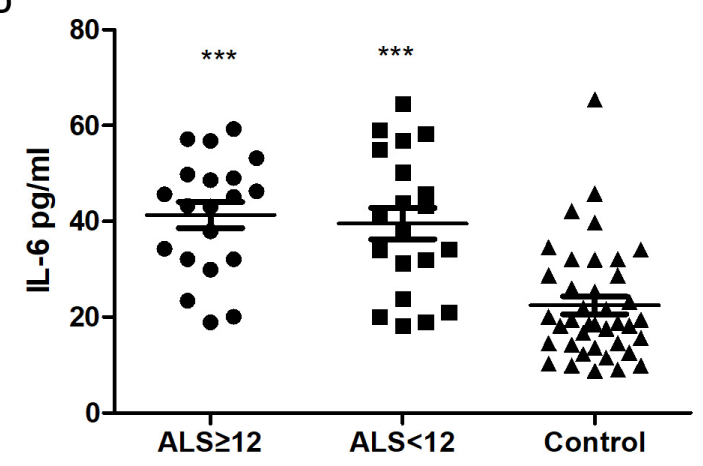

FIGURE 1 | Comparison IL-6 levels in ADEs from plasma of ALS and healthy subjects. Panels (A-D) show the levels of IL-6 in ADEs of (A) ALS patients and controls; (B) ALS patients with bulbar (ALS-B) or limb onset (ALS-L) and controls; (C) definite ALS (ALS-D) or probable ALS (ALS-P) and controls; (D) ALS duration $\geq 12$ months (ALS $\geq 12$ ) or $<12$ months (ALS $<12$ ) and controls. $* *$, *** indicate $p<0.01$ and $p<0.001$, respectively, compared with controls.

pathophysiology of brain disease and the identification of biomarkers (Abner et al., 2016; Winston et al., 2016; Goetzl et al., 2018; Ohmichi et al., 2018). However, to our knowledge, no studies have been reported on CNS-derived exosomes in ALS patients. Therefore, our pilot study is the first to demonstrate that CNS-derived exosomes could be useful to reveal the pathophysiology of CNS in ALS patients.

Several inflammatory biomarkers have been found to be linked to ALS. As a well-known cytokine, IL- 6 has been extensively investigated in neurodegenerative disorders and associated with ALS in numerous studies (Sekizawa et al., 1998; Ehrhart et al., 2015; Lu et al., 2016; Blasco et al., 2017; Hu et al., 2017). However, the results are not consistent across all studies (Moreau et al., 2005; Tanaka et al., 2006). In addition, one study reported an increase in IL-6 levels at the late stage of disease ( $\mathrm{Lu}$ et al., 2016), whereas another study reported that the levels of IL6 were high at disease onset followed by a subsequent decline (Ehrhart et al., 2015). The plasma IL-6 levels were also measured in this study. However, the IL- 6 was detectable in only 12 controls and 15 ALS patients and undetectable in most of the samples. Among the 12 controls and 15 ALS patients, the IL- 6 levels were highly variable and no difference has been found between the two groups. The highly variable plasma IL-6 levels in our study and the contradictory results from previous studies indicate that the peripheral IL-6 levels may be influenced by complex factors. A recent study showed that the levels of IL- 6 in blood could be influenced by aging and respiratory dysfunction in ALS (ProntoLaborinho et al., 2019). Thus, determining the IL-6 levels in blood may not be a good way. CNS-derived exosomes could directly reflect the situation in the CNS, and peripheral factors might have little effect on cytokines in CNS-derived exosomes. Therefore, the measurement of IL-6 levels in CNS-derived exosomes, compared with blood or CSF, may be better to illuminate the actual role of IL-6 in ALS. Astrocytes have been reported play an important role in the pathogenesis of ALS, and the predominant CNS source of IL-6 is the activated astrocyte (Van Wagoner and Benveniste, 1999). Hence, in this pilot study, we chose to measure IL-6 levels in ADEs. Compared with the plasma IL-6 levels, the IL-6 levels in ADEs were relatively high and stable and the IL-6 levels in the ADEs didn't correlate with age. Moreover, it was supposed that there may be connection between IL-6 levels in plasma and ADEs. However, no correlation had been found between two groups. All these results indicate that CNS-derived exosomes may be a promising object to help find biomarkers for ALS.

The important findings of our study were that IL-6 levels in ADEs increased in sALS patients and were positively associated with the rate of disease progression. These data suggest that the IL-6 in ADEs may be a candidate biomarker for ALS. However, neuroinflammation is a common phenomenon in almost all neurological disease. Therefore, it is believed that the IL-6 levels in ADEs probably increase in other neurological conditions. Actually, it has been reported that the IL- 6 levels in ADEs 
A

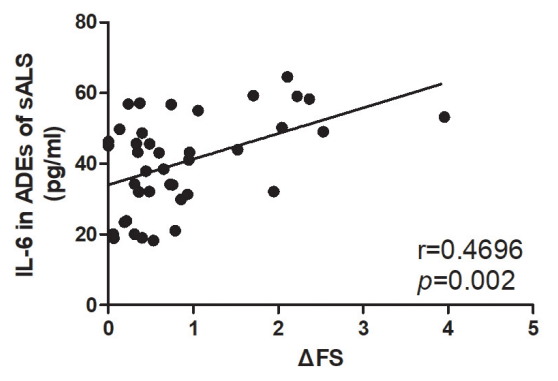

C

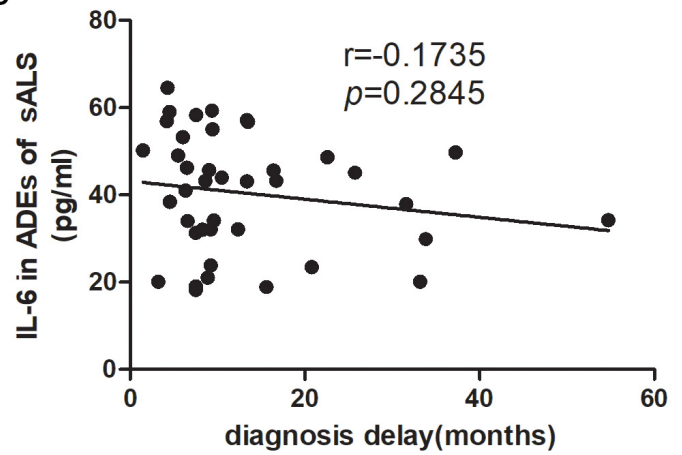

B

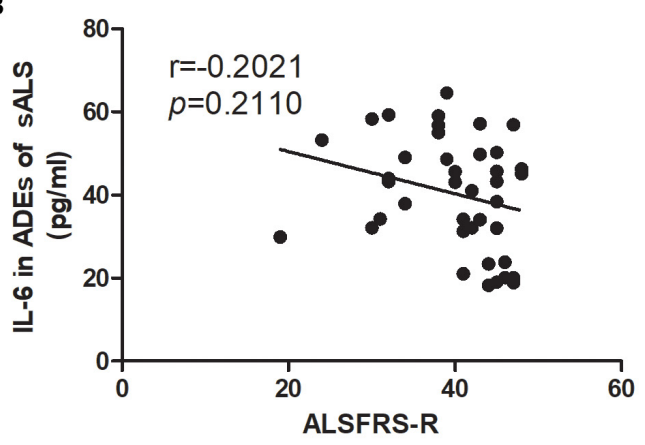

D

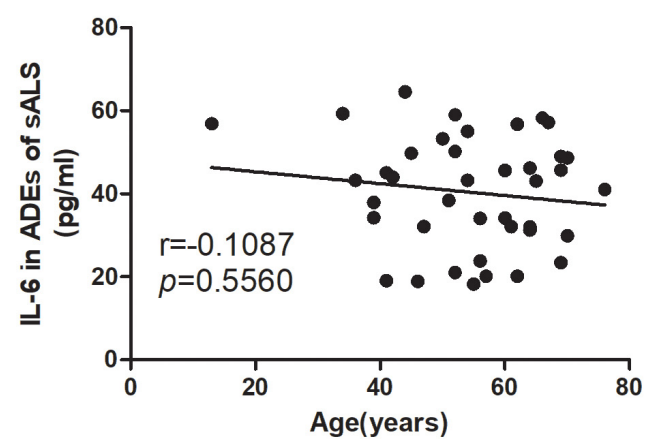

FIGURE 2 | Correlations between IL-6 levels in ADEs of ALS patients with the disease progression rate, ALSFRS-R score, diagnosis delay and patient age. (A) shows that the IL-6 levels in ADEs of ALS patients positively correlate with the disease progression rate. However, the IL-6 levels in ADEs of ALS patients do not correlate with the ALSFRS-R score (B), diagnosis delay (C), and patient age (D).
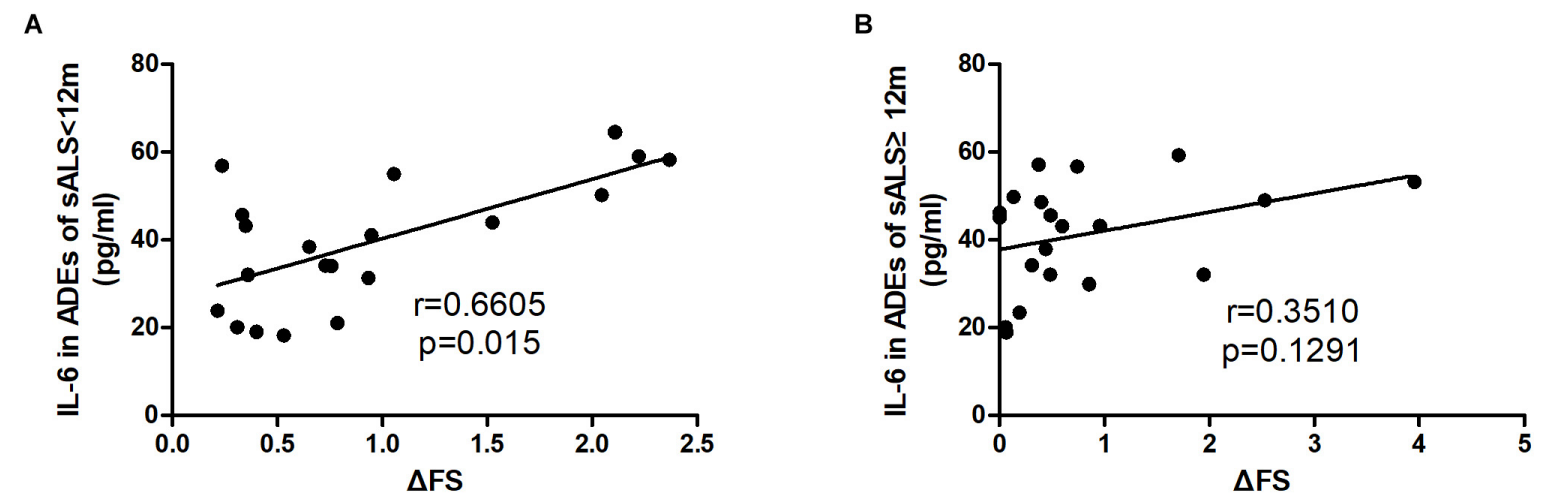

FIGURE 3 | Correlations between IL-6 levels in ADEs of ALS subgroups with the disease progression rate. ALS patients were divided into two groups according to the disease duration. (A) The IL-6 levels in ADEs of the ALS $<12$ group positively correlate with the disease progression rate. However, the II-6 levels in ADEs of the ALS12 group do not correlate with the disease progression rate (B).

increased in AD patients (Goetzl et al., 2018). Thus, the IL-6 levels in ADEs may not be suitable to help discriminate ALS from other neurological diseases. According to our study, measuring the IL6 levels in ADEs may be helpful to reflect the neuroinflammation status and predict disease progression.

We could not determine the precise role of IL-6 in ALS patients because of its complex physiological functions. Increased IL-6 secretion could be a neuroprotective reaction against CNS damage or a pro-inflammatory agent (Spooren et al., 2011).
However, most views consider IL-6 as a pro-inflammatory cytokine in ALS patients. The anti-IL-6 antibody, tocilizumab, has been proposed as a therapeutic drug for ALS (Fiala et al., 2013). Therefore, we speculate that the increase in IL-6 observed in this study was harmful to ALS patients. Our further analyses revealed that the levels of IL-6 did not differ between ALS subgroups, and the correlation between IL- 6 and the rate of disease progression was only observed during the initial 12 months. These results indicated that IL- 6 produced by 
astrocytes might be more important during the early stage of disease. However, our sample size was limited, and the results should therefore be confirmed in further studies.

\section{CONCLUSION}

The present study demonstrated that the levels of IL-6 in ADEs of ALS patients were increased and positively associated with the rate of disease progression, especially in patients at an earlier disease stage. Our pilot study is the first to demonstrate that CNSderived exosomes could be useful to reveal the pathophysiology of CNS in ALS patients.

\section{DATA AVAILABILITY}

The raw data supporting the conclusions of this manuscript will be made available by the authors, without undue reservation, to any qualified researcher.

\section{ETHICS STATEMENT}

This study was approved by the Ethics Committee of the Perking University Third Hospital, Beijing, China. All ALS patients

\section{REFERENCES}

Abner, E. L., Jicha, G. A., Shaw, L. M., Trojanowski, J. Q., and Goetzl, E. J. (2016). Plasma neuronal exosomal levels of Alzheimer's disease biomarkers in normal aging. Ann. Clin. Transl. Neurol. 3, 399-403. doi: 10.1002/acn3.309

Beninson, L. A., and Fleshner, M. (2014). Exosomes: an emerging factor in stressinduced immunomodulation. Semin. Immunol. 26, 394-401. doi: 10.1016/j. smim.2013.12.001

Blasco, H., Garcon, G., Patin, F., Veyrat-Durebex, C., Boyer, J., Devos, D., et al. (2017). Panel of oxidative stress and inflammatory biomarkers in ALS: a pilot study. Can. J. Neurol. Sci. 44, 90-95. doi: 10.1017/cjn.2016.284

Brooks, B. R., Miller, R. G., Swash, M., Munsat, T. L., and World Federation of Neurology Research Group on Motor Neuron Diseases. (2000). El Escorial revisited: revised criteria for the diagnosis of amyotrophic lateral sclerosis, amyotroph lateral scler other motor. Neuron Disord. 1, 293-299. doi: 10.1080/ 146608200300079536

Brown, R. H., and Al-Chalabi, A. (2017). Amyotrophic lateral sclerosis. N. Engl. J. Med. 377, 162-172.

Cedarbaum, J. M., Stambler, N., Malta, E., Fuller, C., Hilt, D., Thurmond, B., et al. (1999). The ALSFRS-R: a revised ALS functional rating scale that incorporates assessments of respiratory function. BDNF ALS study group (Phase III). J. Neurol. Sci. 169, 13-21. doi: 10.1016/s0022-510x(99)00210-5

Chaturvedi, S., Siegel, D., Wagner, C. L., Park, J., van de Velde, H., Vermeulen, J., et al. (2015). Development and validation of panoptic Meso scale discovery assay to quantify total systemic interleukin-6. Br. J. Clin. Pharmacol. 80, 687-697. doi: 10.1111/bcp.12652

Ehrhart, J., Smith, A. J., Kuzmin-Nichols, N., Zesiewicz, T. A., Jahan, I., Shytle, R. D., et al. (2015). Humoral factors in ALS patients during disease progression. J. Neuroinflammation 12:127. doi: 10.1186/s12974-015-0350-4

Fiala, M., Chattopadhay, M., La Cava, A., Tse, E., Liu, G., and Lourenco, E. (2010). IL-17A is increased in the serum and in spinal cord CD8 and mast cells of ALS patients. J. Neuroinflammation 7:76. doi: 10.1186/1742-2094-7-76

Fiala, M., Mizwicki, M. T., Weitzman, R., Magpantay, L., and Nishimoto, N. (2013). Tocilizumab infusion therapy normalizes inflammation in sporadic ALS patients. Am. J. Neurodegener. Dis. 2, 129-139. and age-, sex-matched healthy control individuals signed the informed consent before peripheral blood samples were drawn.

\section{AUTHOR CONTRIBUTIONS}

DF conceived the study, provided the financial support, and responsible for the project management. DF and YC designed the study, responsible for preparing and revising the manuscript, and had key roles in the study. KX and LC took part in the design of the study and in sample collection, and undertook data checking.

\section{FUNDING}

This study was supported by the National Natural Sciences Foundation of China (81873784).

\section{SUPPLEMENTARY MATERIAL}

The Supplementary Material for this article can be found online at: https://www.frontiersin.org/articles/10.3389/fnins. 2019.00574/full\#supplementary-material

Goetzl, E. J., Schwartz, J. B., Abner, E. L., Jicha, G. A., and Kapogiannis, D. (2018). High complement levels in astrocyte-derived exosomes of Alzheimer disease. Ann. Neurol. 83, 544-552. doi: 10.1002/ana.25172

Goh, W. J., Zou, S., Ong, W. Y., Torta, F., Alexandra, A. F., Schiffelers, R. M., et al. (2017). Bioinspired cell-derived nanovesicles versus exosomes as drug delivery systems: a cost-effective alternative. Sci. Rep. 7:14322. doi: 10.1038/s41598-01714725-x

Gonzalez-Garza, M. T., Martinez, H. R., Cruz-Vega, D. E., Hernandez-Torre, M., and Moreno-Cuevas, J. E. (2018). Adipsin. MIP-1b, and IL-8 as CSF biomarker panels for ALS diagnosis. Dis. Markers 2018:3023826. doi: 10.1155/ 2018/3023826

Hu, Y., Cao, C., Qin, X. Y., Yu, Y., Yuan, J., Zhao, Y., et al. (2017). Increased peripheral blood inflammatory cytokine levels in amyotrophic lateral sclerosis: a meta-analysis study. Sci. Rep. 7:9094. doi: 10.1038/s41598-017-09097-1

Italiani, P., Carlesi, C., Giungato, P., Puxeddu, I., Borroni, B., Bossu, P., et al. (2014). Evaluating the levels of interleukin-1 family cytokines in sporadic amyotrophic lateral sclerosis. J. Neuroinflammation 11:94. doi: 10.1186/1742-209411-94

Jarmalaviciute, A., and Pivoriunas, A. (2016). Exosomes as a potential novel therapeutic tools against neurodegenerative diseases. Pharmacol. Res. 113(Pt B), 816-822. doi: 10.1016/j.phrs.2016.02.002

Kimura, F., Fujimura, C., Ishida, S., Nakajima, H., Furutama, D., Uehara, H., et al. (2006). Progression rate of ALSFRS-R at time of diagnosis predicts survival time in ALS. Neurology 66, 265-267. doi: 10.1212/01.wnl.0000194316.91908.8a

Kumar, V., Islam, A., Hassan, M. I., and Ahmad, F. (2016). Therapeutic progress in amyotrophic lateral sclerosis-beginning to learning. Eur. J. Med. Chem. 121, 903-917. doi: 10.1016/j.ejmech.2016.06.017

Kuwano, N., Kato, T. A., Mitsuhashi, M., Sato-Kasai, M., Shimokawa, N., Hayakawa, K., et al. (2018). Neuron-related blood inflammatory markers as an objective evaluation tool for major depressive disorder: an exploratory pilot case-control study. J. Affect Disord. 240, 88-98. doi: 10.1016/j.jad.2018.0 7.040

Liu, J., Gao, L., and Zang, D. (2015). Elevated levels of IFN-gamma in CSF and serum of patients with amyotrophic lateral sclerosis. PLoS One 10:e136937. doi: 10.1371/journal.pone.0136937 
Liu, J., and Wang, F. (2017). Role of neuroinflammation in amyotrophic lateral sclerosis: cellular mechanisms and therapeutic implications. Front. Immunol. 8:1005. doi: 10.3389/fimmu.2017.01005

Lu, C. H., Allen, K., Oei, F., Leoni, E., Kuhle, J., and Tree, T. (2016). Systemic inflammatory response and neuromuscular involvement in amyotrophic lateral sclerosis. Neurol. Neuroimmunol. Neuroinflamm. 3:e244. doi: 10.1212/NXI. 0000000000000244

Mitchell, R. M., Freeman, W. M., Randazzo, W. T., Stephens, H. E., Beard, J. L., Simmons, Z., et al. (2009). A CSF biomarker panel for identification of patients with amyotrophic lateral sclerosis. Neurology 72, 14-19. doi: 10.1212/01.wnl. 0000333251.36681.a5

Mitchell, R. M., Simmons, Z., Beard, J. L., Stephens, H. E., and Connor, J. R. (2010). Plasma biomarkers associated with ALS and their relationship to iron homeostasis. Mus. Nerve 42, 95-103. doi: 10.1002/mus.21625

Moreau, C., Devos, D., Brunaud-Danel, V., Defebvre, L., Perez, T., Destee, A., et al. (2005). Elevated IL-6 and TNF-alpha levels in patients with ALS: inflammation or hypoxia? Neurology 65, 1958-1960. doi: 10.1212/01.wnl.0000188907.973 39.76

Mustapic, M., Eitan, E., Werner, J. K. Jr., Berkowitz, S. T., Lazaropoulos, M. P., Tran, J., et al. (2017). Plasma extracellular vesicles enriched for neuronal origin: a potential window into brain pathologic processes. Front. Neurosci. 11:278. doi: 10.3389/fnins.2017.00278

Ohmichi, T., Mitsuhashi, M., Tatebe, H., Kasai, T., Ali El-Agnaf, O. M., and Tokuda, T. (2018). Quantification of brain-derived extracellular vesicles in plasma as a biomarker to diagnose Parkinson's and related diseases. Parkin. Relat. Disord. 61, 82-87. doi: 10.1016/j.parkreldis.2018.11.021

Ono, S., Hu, J., Shimizu, N., Imai, T., and Nakagawa, H. (2001). Increased interleukin-6 of skin and serum in amyotrophic lateral sclerosis. J. Neurol. Sci. 187, 27-34. doi: 10.1016/s0022-510x(01)00514-7

Pronto-Laborinho, A., Pinto, S., Gromicho, M., Pereira, M., Swash, M., and de Carvalho, M. (2019). Interleukin-6 and amyotrophic lateral sclerosis. J. Neurol. Sci. 398, 50-53. doi: 10.1016/j.jns.2019.01.026

Raposo, G., and Stoorvogel, W. (2013). Extracellular vesicles: exosomes, microvesicles, and friends. J. Cell. Biol. 200, 373-383. doi: 10.1083/jcb. 201211138

Rothstein, J. D. (2017). Edaravone: a new drug approved for ALS. Cell 171:725. doi: 10.1016/j.cell.2017.10.011

Sekizawa, T., Openshaw, H., Ohbo, K., Sugamura, K., Itoyama, Y., and Niland, J. C. (1998). Cerebrospinal fluid interleukin 6 in amyotrophic lateral sclerosis: immunological parameter and comparison with inflammatory and non-inflammatory central nervous system diseases. J. Neurol. Sci. 154, 194-199. doi: 10.1016/s0022-510x(97)00 228-1

Spooren, A., Kolmus, K., Laureys, G., Clinckers, R., De Keyser, J., Haegeman, G., et al. (2011). Interleukin-6, a mental cytokine. Brain Res. Rev. 67, 157-183. doi: 10.1016/j.brainresrev.2011.01.002

Su, X. W., Simmons, Z., Mitchell, R. M., Kong, L., Stephens, H. E., and Connor, J. R. (2013). Biomarker-based predictive models for prognosis in amyotrophic lateral sclerosis. JAMA Neurol. 70, 1505-1511. doi: 10.1001/jamaneurol.2013.4646

Tanaka, M., Kikuchi, H., Ishizu, T., Minohara, M., Osoegawa, M., and Motomura, K. (2006). Intrathecal upregulation of granulocyte colony stimulating factor and its neuroprotective actions on motor neurons in amyotrophic lateral sclerosis. J. Neuropathol. Exp. Neurol. 65, 816-825. doi: 10.1097/01.jnen.0000232025. 84238.e1

Van Wagoner, N. J., and Benveniste, E. N. (1999). Interleukin-6 expression and regulation in astrocytes. J. Neuroimmunol. 100, 124-139. doi: 10.1016/s01655728(99)00187-3

Vu, L. T., and Bowser, R. (2017). Fluid-Based biomarkers for amyotrophic lateral sclerosis. Neurotherapeutics 14, 119-134. doi: 10.1007/s13311-016-0503-x

Winston, C. N., Goetzl, E. J., Akers, J. C., Carter, B. S., Rockenstein, E. M., Galasko, D., et al. (2016). Prediction of conversion from mild cognitive impairment to dementia with neuronally derived blood exosome protein profile. Alzheimers Dement 3, 63-72. doi: 10.1016/j.dadm.2016.04.001

Yanez-Mo, M., Siljander, P. R., Andreu, Z., Zavec, A. B., Borras, F. E., and Buzas, E. I. (2015). Biological properties of extracellular vesicles and their physiological functions. J. Extracell. Vesicles 4:27066. doi: 10.3402/jev.v4.27066

Zhao, W., Beers, D. R., and Appel, S. H. (2013). Immune-mediated mechanisms in the pathoprogression of amyotrophic lateral sclerosis. J. Neuroimmune. Pharmacol. 8, 888-899. doi: 10.1007/s11481-013-9489-x

Conflict of Interest Statement: The authors declare that the research was conducted in the absence of any commercial or financial relationships that could be construed as a potential conflict of interest.

Copyright (c) 2019 Chen, Xia, Chen and Fan. This is an open-access article distributed under the terms of the Creative Commons Attribution License (CC BY). The use, distribution or reproduction in other forums is permitted, provided the original author(s) and the copyright owner(s) are credited and that the original publication in this journal is cited, in accordance with accepted academic practice. No use, distribution or reproduction is permitted which does not comply with these terms. 